Original paper

\title{
Effects of lung tissue characterization in radiotherapy of breast cancer under deep inspiration breath hold when using Monte Carlo dosimetry
}

\author{
P. Andersson ${ }^{\mathrm{a}, \mathrm{c}}$, N. Pettersson ${ }^{\mathrm{b}}$, A. Lindberg ${ }^{\mathrm{b}}$, J. Swanpalmer ${ }^{\mathrm{a}, \mathrm{b}}$, R. Chakarova $^{\mathrm{a}, \mathrm{b},{ }^{*}}$ \\ ${ }^{\text {a }}$ Institute of Clinical Sciences, Department of Medical Radiation Science, Sahlgrenska Academy, University of Gothenburg, Gothenburg, Sweden \\ ${ }^{\mathrm{b}}$ Department of Medical Physics and Biomedical Engineering, Sahlgrenska University Hospital, Gothenburg, Sweden \\ ${ }^{c}$ RISE Research Institutes of Sweden, Materials and Production, Gothenburg, Sweden
}

\section{A R T I C L E I N F O}

\section{Keywords:}

Breast cancer radiotherapy

DIBH

Lung tissue characterization

Absorbed dose

Monte Carlo simulations

\begin{abstract}
A B S T R A C T
Purpose: To investigate the sensitivity of Monte Carlo (MC) calculated lung dose distributions to lung tissue characterization in external beam radiotherapy of breast cancer under Deep Inspiration Breath Hold (DIBH). Methods: EGSnrc based MC software was employed. Mean lung densities for one hundred patients were analysed. CT number frequency and clinical dose distributions were calculated for 15 patients with mean lung density below $0.14 \mathrm{~g} / \mathrm{cm}^{3}$. Lung volume with a pre-defined CT numbers was also considered. Lung tissue was characterized by applying different CT calibrations in the low-density region and air-lung tissue thresholds. Dose impact was estimated by Dose Volume Histogram (DVH) parameters.

Results: Mean lung densities below $0.14 \mathrm{~g} / \mathrm{cm}^{3}$ were found in $10 \%$ of the patients. CT numbers below $-960 \mathrm{HU}$ dominated the CT frequency distributions with a high rate of CT numbers at -990 HU. Mass density conversion approach influenced the DVH shape. $\mathrm{V}_{4 \mathrm{~Gy}}$ and $\mathrm{V}_{8 \mathrm{~Gy}}$ varied by $7 \%$ and $5 \%$ for the selected patients and by $9 \%$ and $3.5 \%$ for the pre-defined lung volume. $\mathrm{V}_{16 \mathrm{~Gy}}$ and $\mathrm{V}_{20 \mathrm{~Gy}}$, were within $2.5 \%$. Regions above 20 Gy were affected. Variations in air- lung tissue differentiation resulted in DVH parameters within 1\%. Threshold at $-990 \mathrm{HU}$ was confirmed by the CT number frequency distributions.

Conclusions: Lung dose distributions were more sensitive to variations in the CT calibration curve below lung (inhale) density than to air-lung tissue differentiation. Low dose regions were mostly affected. The dosimetry effects were found to be potentially important to $10 \%$ of the patients treated under DIBH.
\end{abstract}

\section{Introduction}

An accurate description of the patient geometry in terms of tissue composition and mass density is essential when using the Monte Carlo (MC) method to calculate the absorbed dose to the irradiated volume in radiotherapy. Patient-specific data are extracted from computer tomography (CT) images using the CT calibration curve. The curve correlates CT numbers, expressed in Hounsfield Units (HU), to mass density relative to that of water. It is usually a bilinear function fitting CT numbers obtained after scanning a phantom with inserts of well-known density. The HU scale is furthermore divided into intervals and a predefined tissue or other material is assigned to each interval. The anatomical lung volume includes air, tissue and blood. However, the elemental composition of lung tissue substitute [1] is commonly used in the MC calculations. The CT numbers stored in the patient scan are translated to mass density and material information and patient specific 3D phantoms created. Thus, the CT calibration curve and the material assignment scheme, which are not unique defined, may influence the dose calculations.

A segmentation scheme with four or five HU intervals, (air, lung, soft tissue and bone), has been found to give less accurate MC dose results than segmentation consisting of seven or more intervals [2,3]. Such a refinement usually applied to soft tissue or high-density regions, for example the introduction of multiple bone intervals. The low-density region of the CT calibration curve is defined by air, lung inhale $(0.2$ $\left.\mathrm{g} / \mathrm{cm}^{3}\right)$ and lung exhale $\left(0.5 \mathrm{~g} / \mathrm{cm}^{3}\right)$ tissue equivalent inserts.

However, much lower lung densities than lung inhale appear when using respiratory gating techniques such as deep inspiration breath hold (DIBH) in radiotherapy treatment of breast cancer [4-6]. Investigation of CT datasets acquired during different respiratory phases, has shown

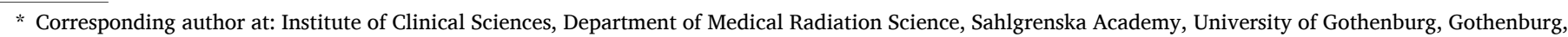
Sweden.

E-mail address: roumiana.chakarova@vgregion.se (R. Chakarova).
} 


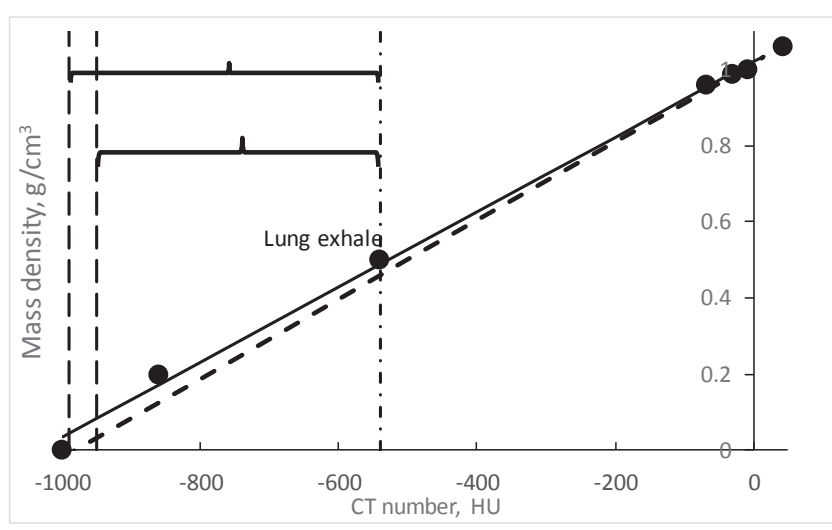

Fig. 1. CT calibration curve below 50 HU. Circles - CT numbers measured in the CIRS phantom inserts; solid line - approximation A; dotted line - approximation B. Brackets indicate the lung tissue assignment for two air-lung tissue boundaries.

well-separated distributions of the mean lung densities: around $0.27 \mathrm{~g} /$ $\mathrm{cm}^{3}$ and $0.16 \mathrm{~g} / \mathrm{cm}^{3}$ for free breathing and DIBH, respectively [7]. Variations in lung density between 0.08 and $0.3 \mathrm{~g} / \mathrm{cm}^{3}$ and between 0.02 and $0.25 \mathrm{~g} / \mathrm{cm}^{3}$ have been reported for DIBH loco-regional and tangential breast treatments, respectively [8]. The CT calibration curve, based on only a few measurement points, is underdefined in this lowdensity region, particularly if the CT response is non-linear.

MC calculations of absorbed dose in low-density lung substitutes have been reported, for example, when evaluating the accuracy of clinical dose calculation algorithms [9-11]. Phantoms were defined by exact geometry and dedicated materials or using an adjusted CT conversion. Tissue characterization based on patient CT images is not so straightforward. It has been shown that mis-assignment of media and/or mass density can lead to significant errors in dose determination [12]. Also, the uncertainty in dose computations significantly increased when real patient geometries were employed. Perturbations applied to the CT calibration for densities larger than lung inhale influenced the MC calculated dose $[13,14]$. The dosimetry impact of possible variations in the CT calibration curve in the low- density region needs further investigation in the case of breast cancer treatment under DIBH.

The importance to correctly distinguish air from lung tissue was illustrated for typical lung inhale and exhale densities [3]. The lung tissue contains hydrogen which is not present in air. Further evaluations are needed in the case of low lung densities observed during DIBH.

The aim of the present study was to investigate the sensitivity of MCcalculated lung dose distributions to lung tissue characterization (density and material assignment) in external beam radiotherapy of breast cancer under DIBH and identify the patient group for which the effects could be important. In particular, we set out to investigate how variations in the conversion of CT numbers to mass density in the low-density region and the choice of air-lung tissue threshold affect the lung dose estimation.

\section{Materials and methods}

MC system based on the EGSnrc software package [15] was used to determine absorbed dose distributions in phantom and patient geometries. The system was initially developed for pre-treatment quality control of volumetric-modulated arc therapy plans generated in the treatment planning system (TPS) Eclipse for 6 MV Varian accelerators, Varian Medical Systems [16]. It was tested for different cancer cites and used clinically in our hospital for independent dose calculations of prostate and gynecological plans in patient geometry. Within this work, the system was further developed to include $15 \mathrm{MV}$ accelerator models to simulate breast cancer treatments involving static multi-leaf collimated $6 \mathrm{MV}$ and $15 \mathrm{MV}$ beams. The phase space file supplied by the vendor was used, describing the radiation field above the secondary collimators. Dose distributions in water were calculated for field sizes between $4 \times 4 \mathrm{~cm}^{2}$ and $20 \times 20 \mathrm{~cm}^{2}$. Lateral profiles at different depths, depth dose profiles and output factors were validated against measurements obtained at accelerator commissioning or for configuration of the clinical treatment planning system. Calibration factor was determined converting MC dose in units Gray per incident particle to Gray per monitor units by relating a simulated to measured dose at the reference geometry. Details about validation procedures, collimator commissioning and absolute dose calculations are given in [17]

Additional comparisons of dose calculations and measurements in large air volume were performed as described below to validate the MC calculations in the extreme case of low-density medium for $6 \mathrm{MV}$ and 15 MV beams.

\subsection{Cylindrical air-filled phantom}

A synthetic diamond detector (PTW microDiamond) with an active volume of $0.004 \mathrm{~mm}^{3}$ was used to determine the absorbed dose in an airfilled cylindrical phantom, $30 \mathrm{~cm}$ in length and $20 \mathrm{~cm}$ in outer diameter with a central air cavity of $17 \mathrm{~cm}$ both in length and diameter. The microDiamond detector was calibrated in a water phantom by using a NE2571 Farmer-type ionization chamber (Nuclear Enterprises, UK). The calibration was completed at our laboratory using a teletherapy unit (TEM Mobaltron, UK) with a ${ }^{60} \mathrm{Co} \gamma$-source. The $\mathrm{N}_{\mathrm{D}, \mathrm{w}}$,Q0 calibration factor for the NE2571 chamber was traceable to a primary standard at the BIPM (Bureau International des Poids et Mesures, France). The calibration coefficient of the microDiamond detector was found to be $1.07 \mathrm{~Gy} / \mathrm{nC}$ in water. Measurements in the air-filled cylindrical phantom were performed for $6 \mathrm{MV}$ and $15 \mathrm{MV}$ photon beams $\left(10 \times 10 \mathrm{~cm}^{2}\right)$ from a Varian True Beam accelerator (schematic view in the insert in Fig. 2a). The microDiamond detector was fixed in a tubular holder with a wall thickness of $0.1 \mathrm{~cm}$ and an inner diameter of $0.7 \mathrm{~mm}$ which was equal to the outer diameter of the detector. The holder was moved orthogonally to the beam axis inside the air cavity in steps of $0.2 \mathrm{~cm}$.

MC calculations were performed for 13 selected measurement points. A CT scan of the cylindrical air-filled phantom was used. The holder at a certain position with the microDiamond detector inside was added as a homogeneous cylinder by modifying the CT images. The material was set to water. In this way, 13 different voxel phantoms were built with a voxel size of $0.15 \times 0.15 \times 0.2 \mathrm{~cm}^{3}$ for independent dose calculations. For each dose calculation $1.10^{9}$ histories were simulated from the phase space produced below jaws for $6 \mathrm{MV}$ and $15 \mathrm{MV}$ photon beams. ECUT was set to $0.521 \mathrm{MeV}$ (including rest mass energy). The continues slowing down approximation (CSDA) range for $0.010 \mathrm{MeV}$ electrons is $2.884 .10^{-4} \mathrm{~g} / \mathrm{cm}^{2}$ [18] which gives $0.24 \mathrm{~cm}$ in dry air. The value is larger than the voxel dimensions in the MC calculations and thus non optimal. However, the cross-section data for lower cutoff were not available in the EGSnrc library. The CPU time to carry out one simulation with eight parallel treads on a Haswell i7 computers was 8-9 h.

The MC results were compared with the experimental data and with dose profiles obtained in Eclipse TPS by the Acuros XB v.15.6 algorithm for the same phantoms as in the MC calculations.

\subsection{Variations in the CT calibration curve and air/lung differentiation}

The patient geometry in the MC calculations was represented by the composition of tissue substitutes [1]: air, lung, adipose, muscle tissues, trabecular and cortical bone. The composition of tree additional bone tissues was refined by interpolation of trabecular and cortical bone following the formalism in [2]. The HU scale was divided into nine discrete intervals with the mass density defined by the selected CT calibration curve. Spencer-Attix restricted mass stopping-power ratios were calculated earlier by the EGSnrc code SPRRZnrc with 6MV Varian accelerator as a source for all the materials used in the MC dose calculations [17]. Corresponding ratios were determined for $15 \mathrm{MV}$ 
(a)

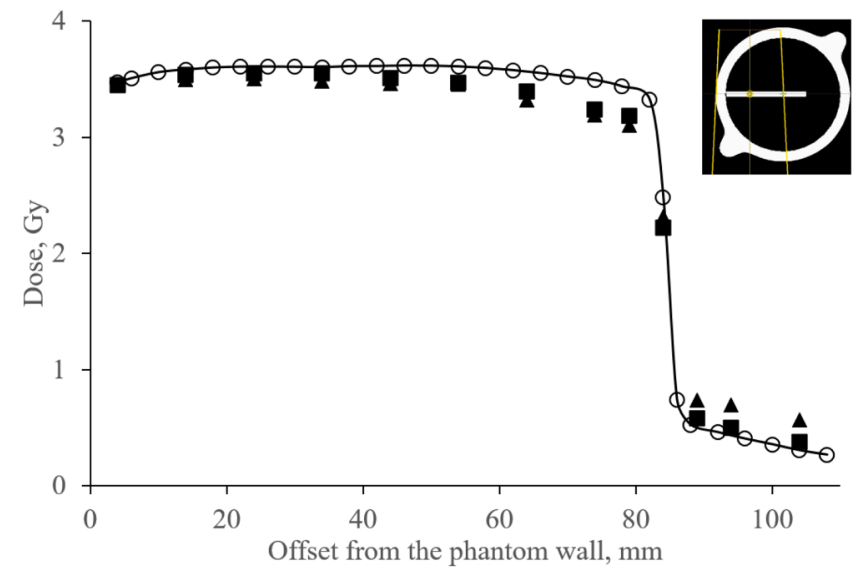

(b)

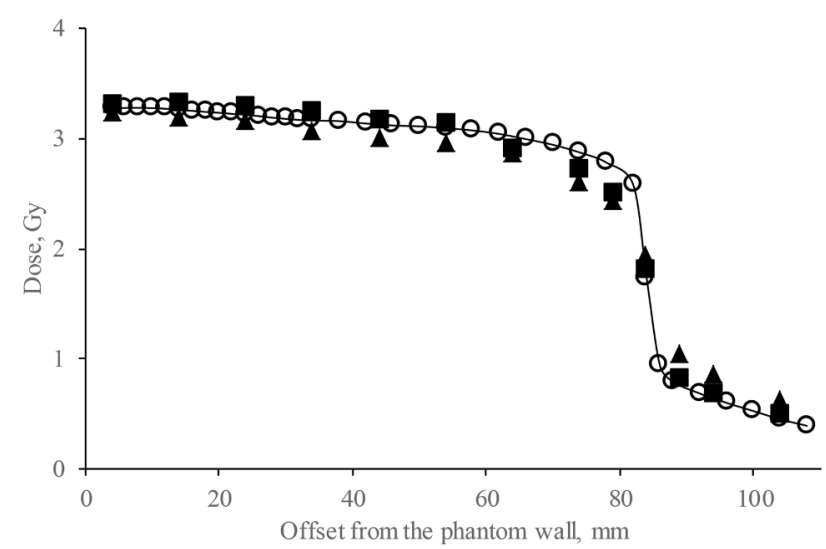

Fig. 2. Measured and calculated values of the absorbed dose profile orthogonal to the beam axis at the isocenter level for (a) the $6 \mathrm{MV}$ beam and (b) the $15 \mathrm{MV}$ beam. Open circles - experimental measurements, boxes - MC data, triangles Acuros XB. Insert in Fig. 2a: transversal view of the phantom with the detector holder in the air cavity at the start position, the irradiation beam indicated.

Table 1

Cases considered for the characterization of the homogeneous lung volume with pre-defined CT numbers of $-990 \mathrm{HU}$.

\begin{tabular}{|c|c|c|c|}
\hline & $\begin{array}{l}\text { Calibration } \\
\text { curve }\end{array}$ & $\begin{array}{l}\text { Lung / air } \\
\text { boundary }\end{array}$ & Lung volume interpretation \\
\hline $\begin{array}{l}\text { MC, scenario } \\
1\end{array}$ & A & $-990 \mathrm{HU}$ & Lung, $0.043 \mathrm{~g} / \mathrm{cm}^{3}$ \\
\hline $\begin{array}{l}\text { MC, scenario } \\
2\end{array}$ & A & $-950 \mathrm{HU}$ & Air, $0.043 \mathrm{~g} / \mathrm{cm}^{3}$ \\
\hline $\begin{array}{l}\text { MC, scenario } \\
\quad 3\end{array}$ & B & $\begin{array}{c}-990 /-950 \\
\text { HU }\end{array}$ & Air, dry air density \\
\hline Acuros XB & A & Overlap & $\begin{array}{l}\text { Air 55\%, Lung 45\%, } 0.015 \\
\mathrm{~g} / \mathrm{cm}^{3}\end{array}$ \\
\hline
\end{tabular}

accelerator beam. The ratios were further implemented to convert the dose in each voxel from dose-to-medium to dose-to-water following the formalism in [19].

A CT calibration curve as a bilinear fitting of measured data was used with a junction point at $50 \mathrm{HU}$. CT numbers were measured in the inserts of a CIRS phantom scanned on a Toshiba Aquilion LB scanner (Toshiba Medical Systems). Measured data below the junction point and the corresponding linear fit, denoted as Approximation A, are shown in Fig. 1. CT numbers of $-1000 \mathrm{HU}$ and below were set to air with dry air mass density. Variation in the CT calibration curve was simulated by assuming a predefined mapping, Approximation B in Fig. 1, used for example by some vendors [20]. The mapping included two different CT numbers for air; outside and inside the patient. In order to investigate the effect of air-lung tissue differentiation, the upper bound of air in the MC segmentation scheme was set to $-990 \mathrm{HU}$ or to $-950 \mathrm{HU}$ (illustrated in Fig. 1), i.e. greater than statistical fluctuations in the image from a modern and an older CT, respectively. No changes were introduced in the differentiation between other tissues.

\subsection{Patient geometry with homogeneous lung volume}

The variations in the CT calibration curve and air/lung differentiation were first implemented to a patient geometry with a homogeneous lung volume as a step towards the real patient geometry. The original CT images of a patient were modified, and CT numbers set to $-990 \mathrm{HU}$ by

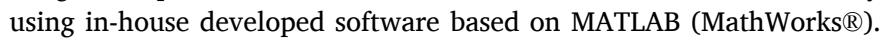
Voxel phantoms were produced applying CT number to mass density conversions A and B and the two values of air-lung tissue boundaries (Table 1). MC dose distributions were obtained for clinical plans involving $6 \mathrm{MV}$ and $15 \mathrm{MV}$ tangential fields. The statistical uncertainty of the voxels occupying the isodose line corresponding to the prescription dose was $0.5 \%$ in the different cases [16] facilitating a stable shape of the dose volume histograms (DVH). The MC results in terms of doseto-medium were compared to each other as well as to the corresponding Eclipse TPS distributions calculated by the algorithm Acuros XB v.15.6.

The measured CT data fitted by approximation A (Fig. 1) were also used in the Acuros XB calculations. However, the range of CT numbers and the material assignments were managed in a different way. For example, Acuros XB allowed an overlapping HU range where two tissues were present in a certain proportion. The lung tissue interpretation by Acuros XB according Eclipse TPS is given in Table 1. The parameters of the DVH used in the comparisons were $\mathrm{V}_{20 G y}, \mathrm{~V}_{16 \mathrm{~Gy}}, \mathrm{~V}_{8 \mathrm{~Gy}}$ and $\mathrm{V}_{4 \mathrm{~Gy}}$, where $V_{X G y}$ denoted the percent of the ipsilateral lung volume receiving at least X Gy, as well as the mean lung dose.

\subsection{Clinical data, non-modified patient geometry}

The mean CT numbers of the ipsilateral lung volumes of 100 consecutively treated patients receiving loco-regional radiation therapy for breast cancer under DIBH were extracted using the structure properties function in Eclipse. These mean CT numbers were compared with corresponding values for 100 consecutively treated patients irradiated under free breathing. All patients were scanned with the same Toshiba Aquilion LB scanner and treated between January and July 2017.

Patients treated under DIBH with a mean CT number below $-890 \mathrm{HU}$ were identified and further analyzed. This CT number is well below the value corresponding to the lung (inhale) insert used in the CT calibration curve. The frequencies of the CT numbers in the lung volumes were mapped with a resolution of $10 \mathrm{HU}$ using an in-house software developed in MATLAB. MC dose calculations were also performed using the clinical plans generated in Eclipse TPS. The plans were based on 3DCRT treatment technique. Anterior-posterior half blocked fields were used to irradiate the lymph nodes complemented with asymmetrical tangential fields for irradiation of the breast tissue. The prescription dose was 50 Gy in 25 fractions. The number of histories simulated was in order of $10^{8}$ and the statistical uncertainty of the voxels occupying the isodose line corresponding to the prescription dose was below $1 \%$ on average. The patient geometry in the MC simulations was defined by $0.25 \times 0.25 \times$ $0.3 \mathrm{~cm}^{3}$ voxels and specified by approximation A or B and an air-lung boundary at $-990 \mathrm{HU}$ or $-950 \mathrm{HU}$.

Dose distributions in terms of dose-to-medium and dose-to-water were compared to each other as well as to the Eclipse results calculated by using Acuros XB v.15.6. The DVH parameters as in the case of the patient geometry with a homogeneous lung volume were monitored in the evaluation. 
(a)

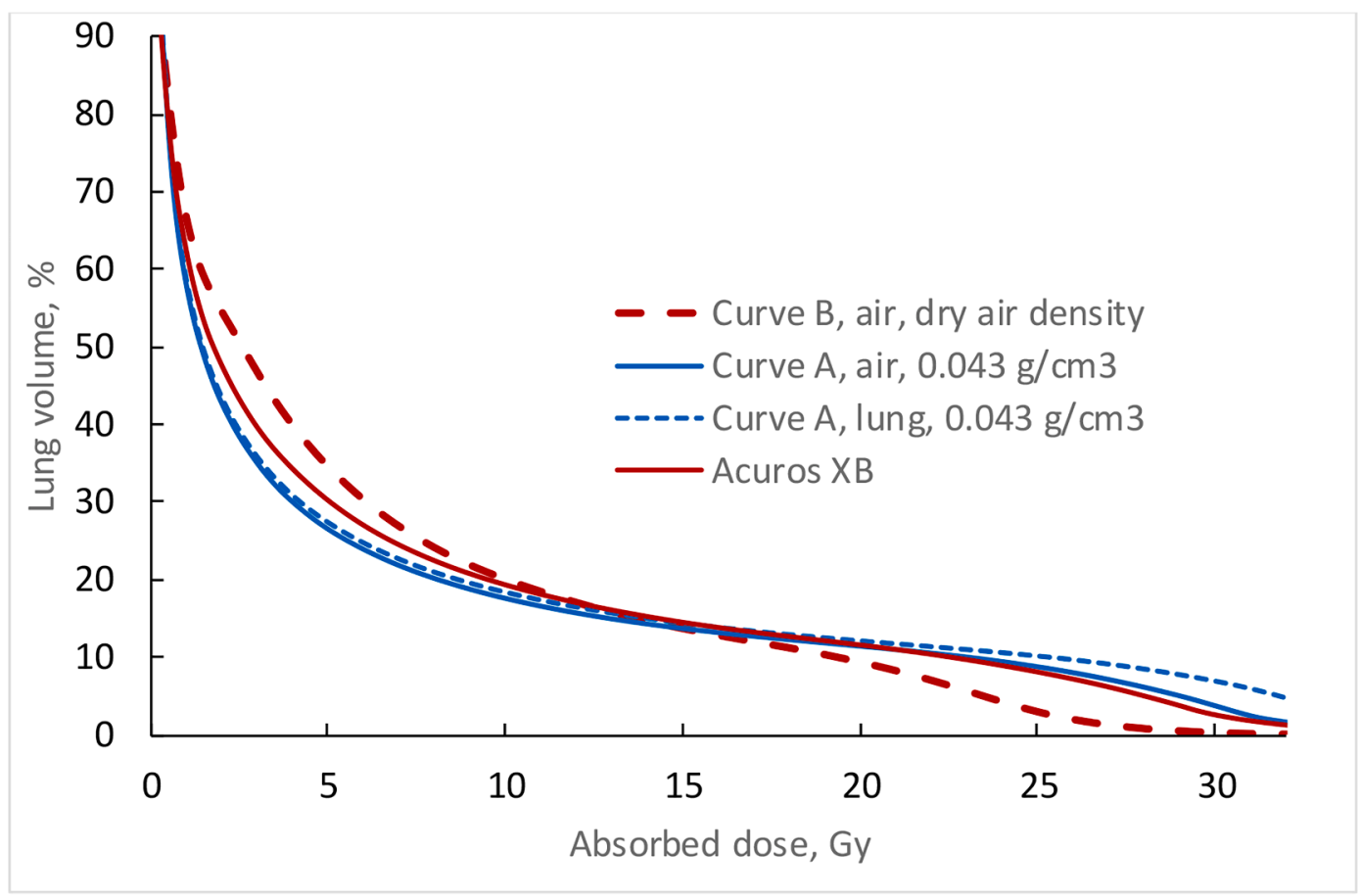

(b)

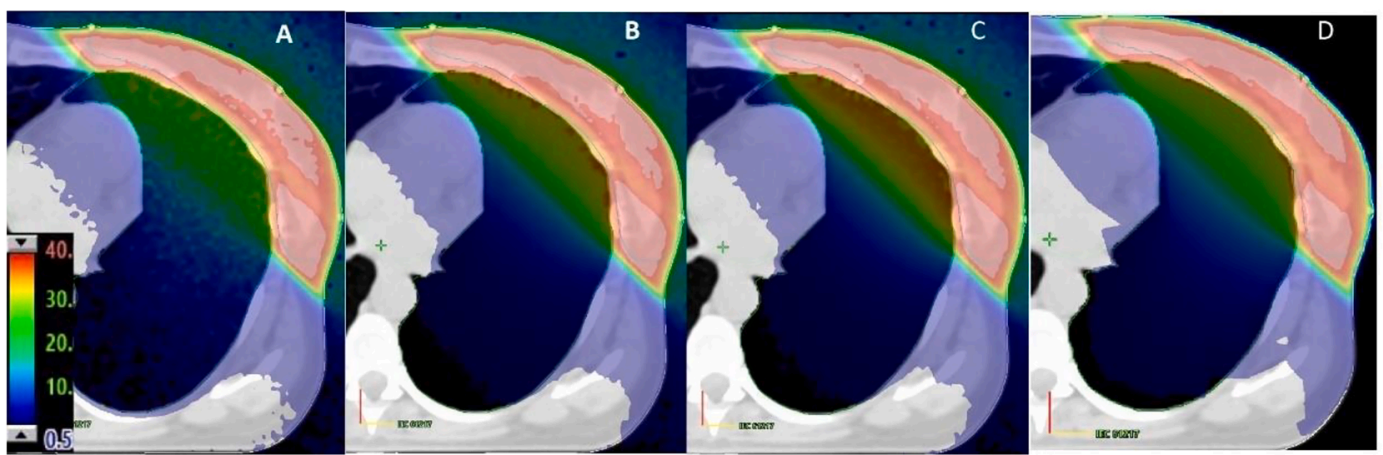

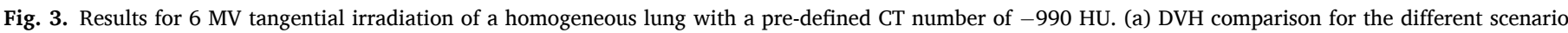

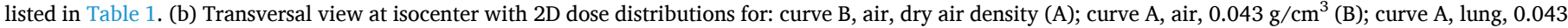
$\mathrm{g} / \mathrm{cm}^{3}$ (C); Acuros XB (D), color scale between $0.5 \mathrm{~Gy}$ and $40 \mathrm{~Gy}$.

\section{Results}

\subsection{Cylindrical air-filled phantom}

Calculated absorbed dose values were compared with experimentally measured data using the microDiamond detector (Fig. 2). The values obtained were not normalized, (i.e. absolute dose comparison). The statistical uncertainty of the computed dose was about $2 \%$ in the high-dose region, up to $5 \%$ in the tail region (not indicated in the figure). The estimated uncertainty in the measurement points was about $1.45 \%$ due to the cross-calibration procedure in ${ }^{60} \mathrm{Co}$ field. Agreement within the uncertainties was found between the experimentally measured and the MC calculated results in the high dose region up to $3.6 \mathrm{~cm}$ from the phantom solid water wall as well as in the tail region. The deviations were larger close to the penumbra. Better agreement was achieved for the $15 \mathrm{MV}$ beam than for the $6 \mathrm{MV}$ beam.
The absorbed dose profile for $6 \mathrm{MV}$, calculated by the Acuros XB algorithm, followed the MC data except in the tail region, where the Acuros XB values were higher. The Acuros XB results for $15 \mathrm{MV}$ were below the experimental and the MC data in the high dose region with the tendency to dominate in the low-dose region outside the beam as for the 6 MV (Fig. 2).

Major factors contributing to the differences found between the calculated and the measured data might be the geometrical and material simplifications of the detector/holder system in the calculations, for example the voxel description of the cylindrical holder assuming homogeneous water material. The lower resolution, i.e. the larger voxels in the MC calculations and in Eclipse TPS than the active volume of the microDiamond detector, may enhance the deviation. 


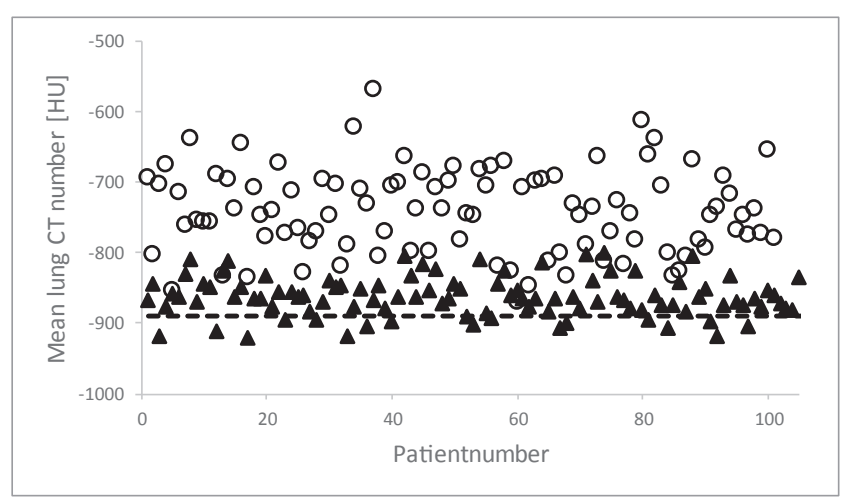

Fig. 4. Mean lung CT number for 100 patients receiving radiation treatment under DIBH (filled triangles) and for 100 patients under free breathing (circles). The dashed line indicates the CT number of $-890 \mathrm{HU}$.

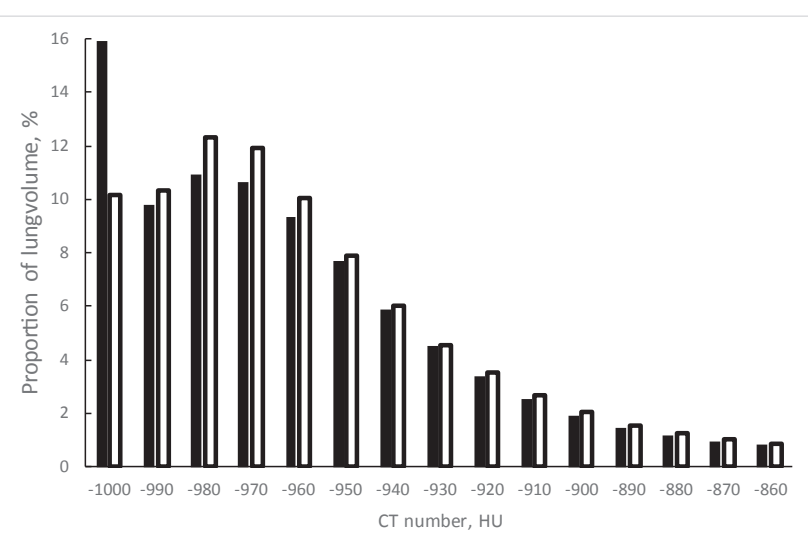

Fig. 5. Frequency distribution of CT numbers in the lung volume for CT scans under DIBH for two patients (black and white bars), $\mathrm{HU}_{\text {mean }}=-909 \mathrm{HU}$ for both scans.

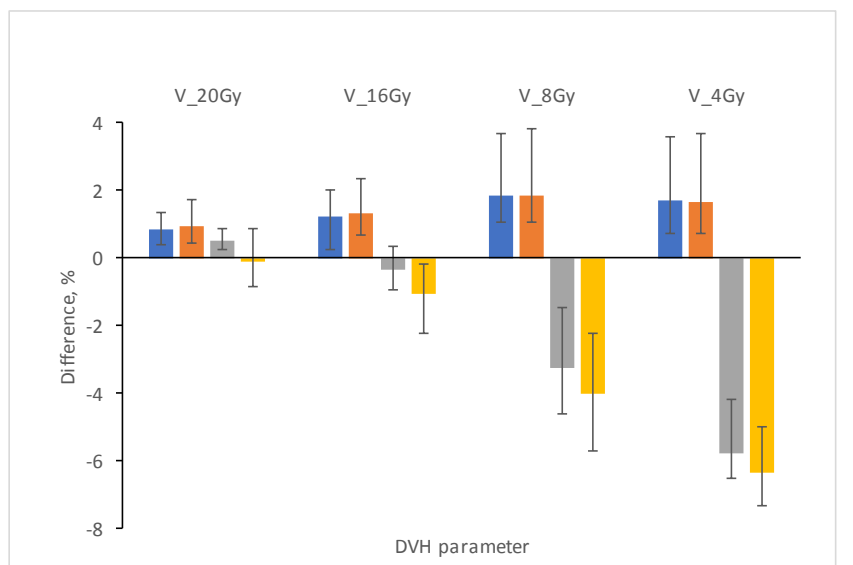

Fig. 6. Deviations (Acuros $\mathrm{XB}$ - MC data) in the average values of the DVH parameters obtained for 15 patients with $\mathrm{HU}_{\text {mean }}$ below $-890 \mathrm{HU}$. Blue and red bars: dose-to-medium and dose-to-water, approximation A in the MC calculations. Grey and yellow bars: dose-to-medium and dose-to-water, approximation $\mathrm{B}$ in the MC calculations. The error bars indicate the minimum and maximum values of the differences found. (For interpretation of the references to color in this figure legend, the reader is referred to the web version of this article.)
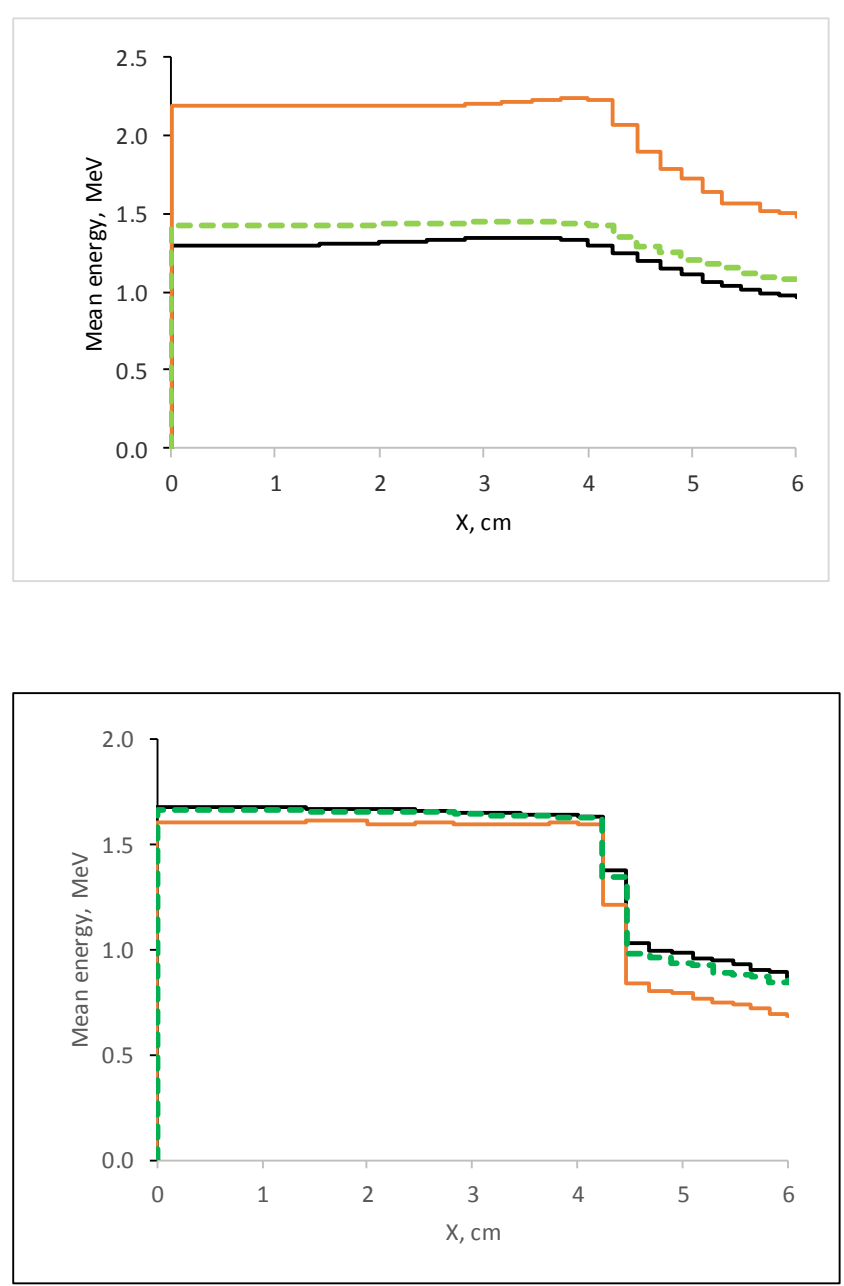

Fig. 1A. Mean energy distribution of electrons (upper plot) and photons (lower plot), transported in air (black), lung tissue composition with a density of 0.05 $\mathrm{g} / \mathrm{cm}^{3}$ (green, dashed) and lung tissue composition with a density of $0.26 \mathrm{~g} / \mathrm{cm}^{3}$ (red). Offset $\mathrm{X}=0 \mathrm{~cm}$ corresponds to the beam axis. (For interpretation of the references to color in this figure legend, the reader is referred to the web version of this article.)

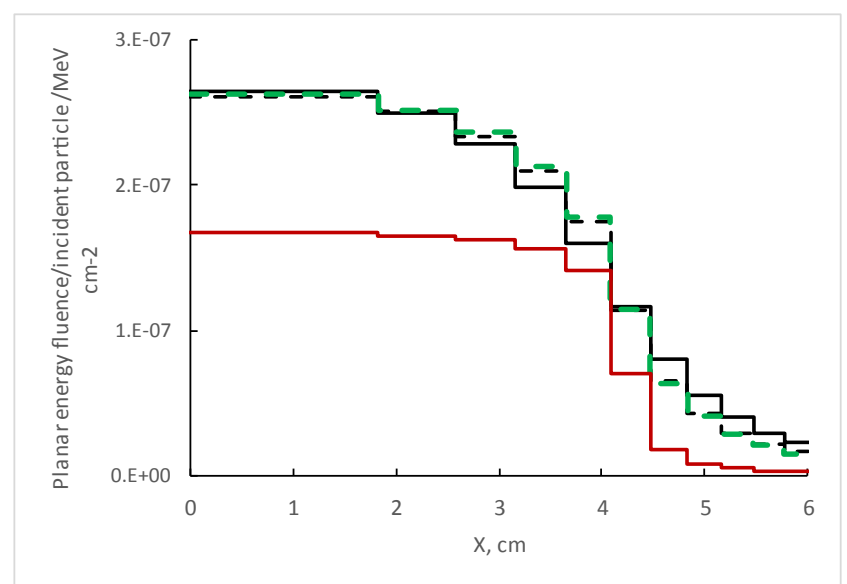

Fig. 2A. Energy fluence vs position for electrons transported in air with dry air density (black), air with $0.05 \mathrm{~g} / \mathrm{cm}^{3}$ density (black, dashed), lung tissue composition with a density of $0.05 \mathrm{~g} / \mathrm{cm}^{3}$ (green dashed) and lung tissue composition with a density of $0.26 \mathrm{~g} / \mathrm{cm}^{3}$ (red). (For interpretation of the references to color in this figure legend, the reader is referred to the web version of this article.) 


\subsection{Patient geometry with homogeneous lung volume}

The MC dose distribution in the lung volume with a pre-defined CT number of $-990 \mathrm{HU}$ was considerably affected by the mass density specification, (illustrated in Fig. 3 for 6 MV beams). Large differences in the DVH were seen between Scenario 1 and 2, (implementation of the CT calibration curve) and Scenario 3 (CT calibration curve B). The DVH parameters $\mathrm{V}_{4 \mathrm{~Gy}}$ and $\mathrm{V}_{8 \mathrm{~Gy}}$ obtained when using the approximation $\mathrm{B}$ were $9.5 \%$ and $3.5 \%$ higher than the corresponding values for approximation A assigning air or lung medium. The DVH parameters were close to each other in the intermediate dose region where maximum deviations within $1 \%$ and $2.5 \%$ were found for $\mathrm{V}_{16 \mathrm{~Gy}}$ and $\mathrm{V}_{20 \mathrm{~Gy}}$, respectively. DVH values corresponding to approximation A were dominating in the high-dose region above $20 \mathrm{~Gy}$.

A similar tendency was found regarding the shape of the DVH for the $15 \mathrm{MV}$ tangential beams showing maximum deviations within $7 \%, 2 \%$, $1.0 \%$ and $2.5 \%$ for $\mathrm{V}_{4 \mathrm{~Gy}}, \mathrm{~V}_{8 \mathrm{~Gy}}, \mathrm{~V}_{16 \mathrm{~Gy}}$ and $\mathrm{V}_{20 \mathrm{~Gy}}$, respectively. The mean doses to the lung agreed within $1 \%$ in both cases, $6 \mathrm{MV}$ and $15 \mathrm{MV}$.

The influence of the material specification, air or lung with the same density of $0.043 \mathrm{~g} / \mathrm{cm}^{3}$, was small with some differences in the dose region above $25 \mathrm{~Gy}$, (curve A for air and for lung in Fig. 3). The DVH parameters were within $1 \%$. Radiation transport of a $6 \mathrm{MV}$ beam in lung tissue with $0.05 \mathrm{~g} / \mathrm{cm}^{3}$ density and in air medium showed similar characteristics (analysis presented in Appendix).

The DVH obtained by Acuros XB were closer to these calculated by $\mathrm{MC}$ for lung or air medium with the mass density of $0.043 \mathrm{~g} / \mathrm{cm} 3$, than to the DVH corresponding to the dry air density (Fig. 3a). The deviation in the high-dose region between the DVH obtained by Acuros XB and by $\mathrm{MC}$, using curve A and lung tissue, was not reflected in the DVH parameters chosen in the evaluation. It was seen, however, when comparing the corresponding transversal 2D dose distributions (plot $\mathrm{C}$ and D in Fig. 3b)

\subsection{Patient geometry with non-modified patient CT data}

The mean CT numbers of the ipsilateral lung volume, ( $\left.\mathrm{HU}_{\text {mean }}\right)$, obtained for the free-breathing scans of the patients were in the range of -871 to $-569 \mathrm{HU}$ (Fig. 4). The average value of $-742 \mathrm{HU}$ corresponded to a mass density of 0.25 and $0.29 \mathrm{~g} / \mathrm{cm}^{3}$ when applying the approximations A and B of the CT calibration curve, respectively. The interval of the $\mathrm{HU}_{\text {mean }}$ for the lung volumes defined by CT scans under DIBH, was $-938 \mathrm{HU}$ to $-801 \mathrm{HU}$. The average value of $-864 \mathrm{HU}$ corresponded to a mass densitiy of $0.12 \mathrm{~g} / \mathrm{cm}^{3}$ and $0.17 \mathrm{~g} / \mathrm{cm}^{3}$ in the approximations $A$ and $\mathrm{B}$, respectively. The densities obtained in the present investigation were comparable to those reported in [7]. Values of $\mathrm{HU}_{\text {mean }}$ below $-890 \mathrm{HU}$ were found for $10 \%$ of the patients treated under DIBH (below the dashed line in Fig. 4). This group of 10 patients was further investigated. Five additional patients were included to improve the statistics of the results.

The assessment of $\mathrm{HU}_{\text {mean }}$ provided a general information related to the density of the whole lung. However, different regions of the lung were found to exhibit different properties. For example, the mean CT numbers for the lung volumes encompassed by the $4 \mathrm{~Gy}$ isodose level defined in the clinical dose distribution in Eclipse were systematically 10 to $25 \mathrm{HU}$ lower than the $\mathrm{HU}_{\text {mean }}$. The frequency distribution of the CT numbers in the whole lung volume exhibited different features for different patients and it was also the case for similar $\mathrm{HU}_{\text {mean }}$ values. Typical frequency distributions contained a high rate of CT numbers less than $-990 \mathrm{HU}$ and CT numbers below $-960 \mathrm{HU}$ dominated (Fig. 5). These findings confirmed a large presence of lung densities well below the lung (inhale) in lung volumes under DIBH.

On average, the DVH parameters $\mathrm{V}_{4 \mathrm{~Gy}}$ and $\mathrm{V}_{8 \mathrm{~Gy}}$ were $7 \%$ and $5 \%$ higher for lung density characterization by approximation $B$ than those using approximation A. These results were in accordance with the findings for a homogeneous lung with a pre-defined CT number of -990 HU. The values of $\mathrm{V}_{16 \mathrm{~Gy}}$ and $\mathrm{V}_{20 \mathrm{~Gy}}$ agreed within $1.5 \%$ and $1 \%$, respectively, for dose calculations in terms of dose to medium. These deviations increased by about $1 \%$ when the dose-to-water was calculated.

The results from the comparison between MC and Acuros XB data depended on the lung tissue characterization used in the MC calculations (Fig. 6). The DVH parameters $\mathrm{V}_{16 \mathrm{~Gy}}, \mathrm{~V}_{8 \mathrm{~Gy}}$ and $\mathrm{V}_{4 \mathrm{~Gy}}$, obtained by Acuros $\mathrm{XB}$ were systematically higher or lower than the corresponding $\mathrm{MC}$ parameters, calculated when using approximation A or B of the CT calibration curve, respectively. The results were following the observations in the case of patient geometry with homogeneous lung volume (Fig. 3a). Opposite differences were expected for parameters related to doses larger than $20 \mathrm{~Gy}$ as the figure shows. Such parameters are usually not of clinical interest and were not explicitly evaluated.

The deviations in the dose-to-water comparison were larger than those in terms of dose-to-medium, more pronounced for approximation $\mathrm{B}$, (discussed further in the next section). In general, the retrospective conversion from dose-to-medium to dose-to-water is known to increase the final uncertainty in the dose determination due to the difference between electron fluence distributions which is not corrected for in the common conversion procedure [21]

\section{Discussion}

Variations in the conversion of CT numbers to mass density below lung (inhale) density were seen to potentially influence the MC dose distributions in the ipsilateral lung for $10 \%$ of the patients treated under DIBH. The effect was larger in the low dose region, $\left(\mathrm{V}_{4 \mathrm{~Gy}}, \mathrm{~V}_{8 \mathrm{~Gy}}\right)$. The clinical plans in this work were based on the 3DCRT treatment technique. Different irradiation techniques, for example volumetric modulated arc therapy, would cause large lung volumes to receive low doses, thus enhancing the sensitivity of the MC dose determination to variations in the CT calibration curve.

A bilinear fitting of measured CT numbers may introduce uncertainties in the mass density conversion for a non-linear CT response. Representation of the low-density region of the CT calibration curve by multi-linear segments would improve the analytical description. This approach requires, however, a high reliability in the measured points defined by the different inserts. Possible uncertainties in the technical specifications of the tissue equivalent phantom materials may propagate to the tissue characterization and, consequently, to dose determination steps. For example, a calculated lung (inhale) density of $0.179 \mathrm{~g} / \mathrm{cm}^{3}$ for a CIRS phantom insert has been reported, whereas the density value given by the manufacturer was $0.195 \mathrm{~g} / \mathrm{cm}^{3}$ [22]. The use of inserts with a lung composition and a mass density between that of air and the conventional lung (inhale) inserts may provide more information on the CT response. In general, the CT numbers obtained for a phantom on a certain CT scanner may be dependent on the reconstruction algorithm, the processing filters and other acquisition parameters [23-25]. Thus, the effect of perturbations in the CT calibration curve on the MC dose calculation is of importance.

The sensitivity of the lung dose to variations in the boundary between the low-density lung tissue and air was found to be low, within the statistical uncertainty of the dose determination. Differentiation at -990 HU could be explained by the results from the frequency analysis of the CT numbers in lung volumes in cases of DIBH. This setting of the air-lung tissue boundary may lead to assignment of lung tissue to air cavities inside the patient geometry. No erroneous assignment was observed when investigating the trachea region in several patient geometries. In general, different thresholds of material differentiation can be set for the different irradiated regions in the body [26].

The absorbed dose in air volumes inside the body, (for example cavities in the head and neck region and trachea), is usually not of clinical interest. However, a high frequency of CT numbers corresponding to air may be present in the lung volume under DIBH. Hence, theoretical and experimental investigation of the absorbed dose profile in the cylindrical air-filled phantom is of great interest. It should be 
noted that experimental measurements in air cavities are a challenging task. Most of the detectors available are not suitable for these measurements because of their energy-, dose rate dependencies, low signal and low spatial resolution. The signal from a microDiamond detector is independent of the beam energy and dose rate. Also, the detector signal is relatively high despite the small active volume of this detector.

Determination of the absorbed dose in air volumes is also important for clinical dose calculation algorithms since the low-density lung tissue may be presented as an air-lung mixture. For example, including air in the material library of Acuros XB was one of the modifications that improved the accuracy of the absorbed dose calculation for tumors located in the esophagus and also in cases of inhomogeneous geometries with low-density (light) lung [9,27].

Acuros XB algorithm uses an energy-dependent fluence-to-dose response function based on the material properties of the voxel for doseto-medium calculation or based on water for dose-to-water estimation. Stopping power ratios are implemented by the MC method. Both, the energy deposition, and the stopping power water/lung ratios are close to unity with small differences in the low-energy region [28]. Much larger ratios apply for air (stopping power ratios above 1.1). Lung volumes with low density described by Acuros XB and the MC method may contain different numbers of air and lung tissue voxels which may enhance the differences in the calculated dose in terms of both, dose-tomedium and dose-to-water.

Acuros XB employs electron cutoff of $0.500 \mathrm{MeV}$ kinetic energy [28] with a CSDA range of $1.8 \mathrm{~cm}$ in dry air [18]. The value is larger than the cutoffs of $0.01 \mathrm{MeV}$ or $0.189 \mathrm{MeV}$ (kinetic energy) typically applied in the MC calculations. How the implementation of different cutoff influences the dose estimation in the presence of low-density voxels is an important issue outside the present work.

\section{Conclusions}

MC dose distributions in lung volumes with mean density below $0.14 \mathrm{~g} / \mathrm{cm}^{3}$ were sensitive to differences in the conversions of CT number to mass density in the air-lung inhale region. The shape of the DVH was influenced in both the low and the high-dose regions. The effect was stronger in the low dose region. Deviations up to $9 \%$ were found between the $V_{4 G y}$ parameters for a lung volume, specified as air with dry air density or specified as air or lung tissue with a density of $0.043 \mathrm{~g} / \mathrm{cm}^{3}$, for clinical plans based on 3DCRT treatment technique. Enhanced sensitivity would be expected for irradiation techniques resulting in low dose to large lung volumes.

Variations in the differentiations between air and the low-density lung tissue resulted in DVH parameters within 1\%. Air-lung tissue threshold at $-990 \mathrm{HU}$ was justified by CT number frequency distribution in lung volumes obtained by CT scans under DIBH. The dosimetry effects were found to be potentially relevant to $10 \%$ of the patients treated under DIBH.

\section{Declaration of Competing Interest}

The authors declare that they have no known competing financial interests or personal relationships that could have appeared to influence the work reported in this paper.

\section{Acknowledgements}

Financial support of King Gustav the V Jubilee Clinic Foundation, The Swedish Radiation Safety Authority and The Healthcare Committee, Region Västra Götaland are greatly acknowledged.

\section{Appendix}

\section{Radiation field characteristic in air and a low-density lung tissue}

\section{Phantom slab geometry}

To better understand the effect of both density and composition on the dose deposition, radiation transport was simulated in a slab geometry consisting of a $2 \mathrm{~cm}$ water slab followed by an $8 \mathrm{~cm}$ slab. The $8 \mathrm{~cm}$ slab was defined in different ways: as air with the density of dry air or $0.05 \mathrm{~g} / \mathrm{cm}^{3}$ or defined as an ICRU lung with a mass density of $0.05 \mathrm{~g} / \mathrm{cm}^{3}$ or $0.26 \mathrm{~g} / \mathrm{cm}^{3}$. Monte Carlo simulations were performed using a normally incident $6 \mathrm{MV}$ photon beam. Phase space files, containing the characteristics of the particles crossing an imaginary plane inside the air or the lung slab were collected at a depth of $5 \mathrm{~cm}$. The files were further analyzed by using the EGSnrc beamdp tool to obtain photon- and electron angular distributions, as well as spectral and mean energy distributions.

The phase space characteristics of the photons and electrons collected in a lung tissue composition with a density of $0.05 \mathrm{~g} / \mathrm{cm}^{3}$ were found to be close to those in air with a density of dry air and in air with a density of $0.05 \mathrm{~g} / \mathrm{cm}^{3}$. Photon and electron characteristics are presented in Figs. $1 \mathrm{~A}$ and 2A. Histograms corresponding to radiation penetrating lung tissue composition with lung-inhale density, $(0.26 \mathrm{~g} / \mathrm{cm} 3)$, are given for comparison, the histogram for air with a density of $0.05 \mathrm{~g} / \mathrm{cm}^{3}$ not shown. The electron transport was more affected by the material composition and density than the photon transport. The differences in the mean energy of photons were mostly outside the radiation field.

The phase space analysis in the case of slab geometry showed that the composition effect, (air or lung tissue composition with very low density), was smaller than the density effect (very low vs lung-inhale density). This analysis provided a better understanding of the low sensitivity of the MC dose on the air - lung tissue differentiation for a lung tissue with mass densities in cases of DIBH.

\section{References}

[1] Tissue substitutes in radiation dosimetry and measurement Report 44 (Bethesda, MD: International Commission on Radiation Units and Measurements) ICRU 1989.

[2] Schneider W, Bortfeld T, Schlegel W. Correlation between CT numbers and tissue parameters needed for Monte Carlo simulations of clinical dose distributions. Phys Med Biol 2000;45(2):459-78.

[3] Vanderstraeten B, Chin PW, Fix M, Leal A, Mora G, Reynaert N, et al. Conversion of CT numbers into tissue parameters for Monte Carlo dose calculations: a multicentre study. Phys Med Biol 2007;52(3):539-62. https://doi.org/10.1088/00319155/52/3/001.

[4] Latty D, Stuart KE, Wang W, Ahern V. Review of deep inspiration breath-hold techniques for the treatment of breast cancer. J Med Radiat Sci 2015;62:74-81. https://doi.org/10.1002/jmrs.

[5] Pandeli C, Smyth LML, David S, See AW. Dose reduction to organs at risk with deep inspiration breath-hold during right breast radiotherapy: a treatment planning study. Radiat Onc 2019;14:223. https://doi.org/10.1186/s13014-019-1430-x.

[6] Haussmann J, Corradini S, Nestle-Kraemling C, Bölke E, Njanang FJD, Tamaskovics B, et al. Recent advances in radiotherapy of breast Cancer. Radiat Onc 2020;15(1). https://doi.org/10.1186/s13014-020-01501-x.

[7] Fogliata A, Nicolini G, Vanetti E, Clivio A, Winkler P, Cozzi L. E Vanetti E, Clivio A, Winkler P, Cozzi L. The impact of photon dose calculation algorithms on expected dose distributions in lungs under different respiratory phases Phys Med Biol 2008; 53(9):2375-90.

[8] Hedin E, Bäck A, Chakarova R. Impact of lung density on the lung dose estimation for radiotherapy of breast cancer. Phys Imaging Radiat Onc (PHIRO) 2017;3:5-10.

[9] Fogliata A, Nicolini G, Clivio A, Vanetti E, Cozzi L. Dosimetric evaluation of Acuros XB Advanced Dose Calculation algorithm in heterogeneous media. Radiat Onc 2011;6:1-15. http://www.ro-journal.com/content/6/1/82.

[10] Beilla S, Younes T, Vieillevigne L, Bardies M, Franceries X, Simon L. Monte Carlo dose calculation in presence of low-density media: Application to lung SBRT treated during DIBH. Phys Med 2017;41:46-52.

[11] Aarup LR, Nahum AE, Zacharatou C, Juhler-Nøttrup T, Knöös T, Nyström H, et al. The effect of different lung densities on the accuracy of various radiotherapy dose calculation methods: Implications for tumour coverage. Radiother Onc 2009;91(3): 405-14.

[12] Verhaegen F, Devic S. Sensitivity study for CT image use in Monte Carlo treatment planning. Phys Med Biol 2005;50(5):937-46. 
[13] Fang R, Mazur T, Mutic S, Khan R. The impact of mass density variations on an electron Monte Carlo algorithm for radiotherapy dose calculations. Phys and Imag Radiat Onc (phiRO) 2018;8:1-7.

[14] Nakao M, Ozawa S, Yogo K, Miura H, Yamada K, Hosono F, et al. Tolerance levels of mass density for CT number calibration in photon radiation therapy. J Appl Clin Med Phys 2019;20(6):45-52. https://doi.org/10.1002/acm2.2019.20.issue$610.1002 / \mathrm{acm} 2.12601$.

[15] Kawrakow I, Mainegra-Hing E, Rogers D W O, Tessier F, Walters B R B. The EGSnrc Code System: Monte Carlo simulation of electron and photon transport. Technical Report PIRS-701, National Research Council Canada 2000 https://nrc-cnrc.github. io/EGSnrc/doc/pirs701-egsnrc.pdf.

[16] Chakarova R, Cronholm R, Krantz M, Andersson P, Hallqvist A. An automated Monte Carlo QC system for volumetric modulated arc therapy: possibilities and challenges. Phys Med 2018;51:32-7.

[17] Chakarova R, Cronholm R, Andersson P, Krantz M. Monte Carlo patient-specific pretreatment QA system for volumetric modulated arc therapy. Report 2017:13 ISSN 2000-0456,. available at www.stralsakerhetsmyndigheten.se/publikationer.

[18] Berger MJ, Coursey JS , Zucker MA and Chang J, Seltzer SM, Bergstrom PM. NIST Standard Reference Database 124, https://dx.doi.org/10.18434/T4NC7P, available at https://www.nist.gov/pml/stopping-power-range-tables-electronsprotons-and-helium-ions.

[19] Siebers V, Keall PJ, Nahum AE, Mohan R. Converting absorbed dose to medium to absorbed dose to water for Monte Carlo based beam dose calculations. Phys Med Biol 2000;45:983-95.

[20] Richmond N. The accuracy of treatment planning system dose modelling in the presence of brass mesh bolus. Reports of Practical Onc Radiother 2017;22(5): 354-9.

[21] Andreo P. Dose to 'water-like' media or dose to tissue in MV photons radiotherapy treatment planning: still a matter of debate. Phys Med Biol 2015; 60: 309-337. Corrigendum p. 2619.
[22] Gustafsson M, Pettersson E, Thilander-Klang A. Validation of Proton Stopping Power Ratios for Tissue Surrogates in an Electron Density Phantom 2017 Int. J. Particle Therapy, Additional Proceedings 56th Annual Meeting of the Particle Therapy Cooperative Group (PTCOG), PTC17-0130 doi:10.14338/IJPT.17-PTCOG2.1 .

[23] Das IJ, Cheng CW, Cao M, Johnstone PAS. Computed tomography imaging parameters for inhomogeneity correction in radiation treatment planning. J Med Phys 2016;41:3-11. https://doi.org/10.4103/0971-6203.177277: 10.4103/0971 6203.177277.

[24] Davis AT, Palmer AL, Nisbet A. Can CT scan protocols used for radiotherapy treatment planning be adjusted to optimize image quality and patient dose? A systematic review. Br J Radiol 2017;90(1076):20160406. https://doi.org/ 10.1259/bjr.20160406.

[25] Davis AT, Palmer AL, Pani S, Nisbet A. Assessment of the variation in CT scanner performance (image quality and Hounsfield units) with scan parameters, for image optimisation in radiotherapy treatment planning. Phys Med 2018;45:59-64. https://doi.org/10.1016/j.ejmp.2017.11.036.

[26] Ottosson RO, Behrens CF. CTC-ask: a new algorithm for conversion of CT numbers to tissue parameters for Monte Carlo dose calculations applying DICOM RS knowledge. Phys Med Biol 2011;56(22):N263-74. https://doi.org/10.1088/00319155/56/22/N01.

[27] Ojala J, Kapanen M. Quantification of dose differences between two versions of Acuros XB algorithm compared to Monte Carlo simulations - the effect on clinical patient treatment planning. J of Appl Clin Med Phys 2015;16(6):213-25.

[28] Failla G A, Wareining T, Archambault Y, Thompson S. Acuros XB advanced dose calculation for the Eclipse treatment planning system. Varian medical systems. Palo Alto California. Clinical perspectives. Acuros XB, available at https://varian. widen.net/view/pdf/xo1pmzdzjb/Acuros_ClinicalPerspectives_RAD10156A_ April2015.pdf?u=wefire. 